\title{
Selective Non-Methylated CpG DNA Recognition Mechanism of Cysteine Clamp Domains
}

Bo Duan ${ }^{1}$, Dihong Fu ${ }^{1}$, Chaoqun Zhang ${ }^{1}$, Pengfei Ding ${ }^{1}$, Xianzhi Dong ${ }^{2 *}$, Bin Xia ${ }^{1 *}$

${ }^{1}$ Beijing Nuclear Magnetic Resonance Center, College of Chemistry and Molecular Engineering, and School of Life Sciences, Peking University, Beijing 100871, China;

${ }^{2}$ Institute of Biophysics, Chinese Academy of Science, Beijing, 100101, China

KEYWORDS : CpG methylation, C-clamp, GEF, TCF, zinc finger 


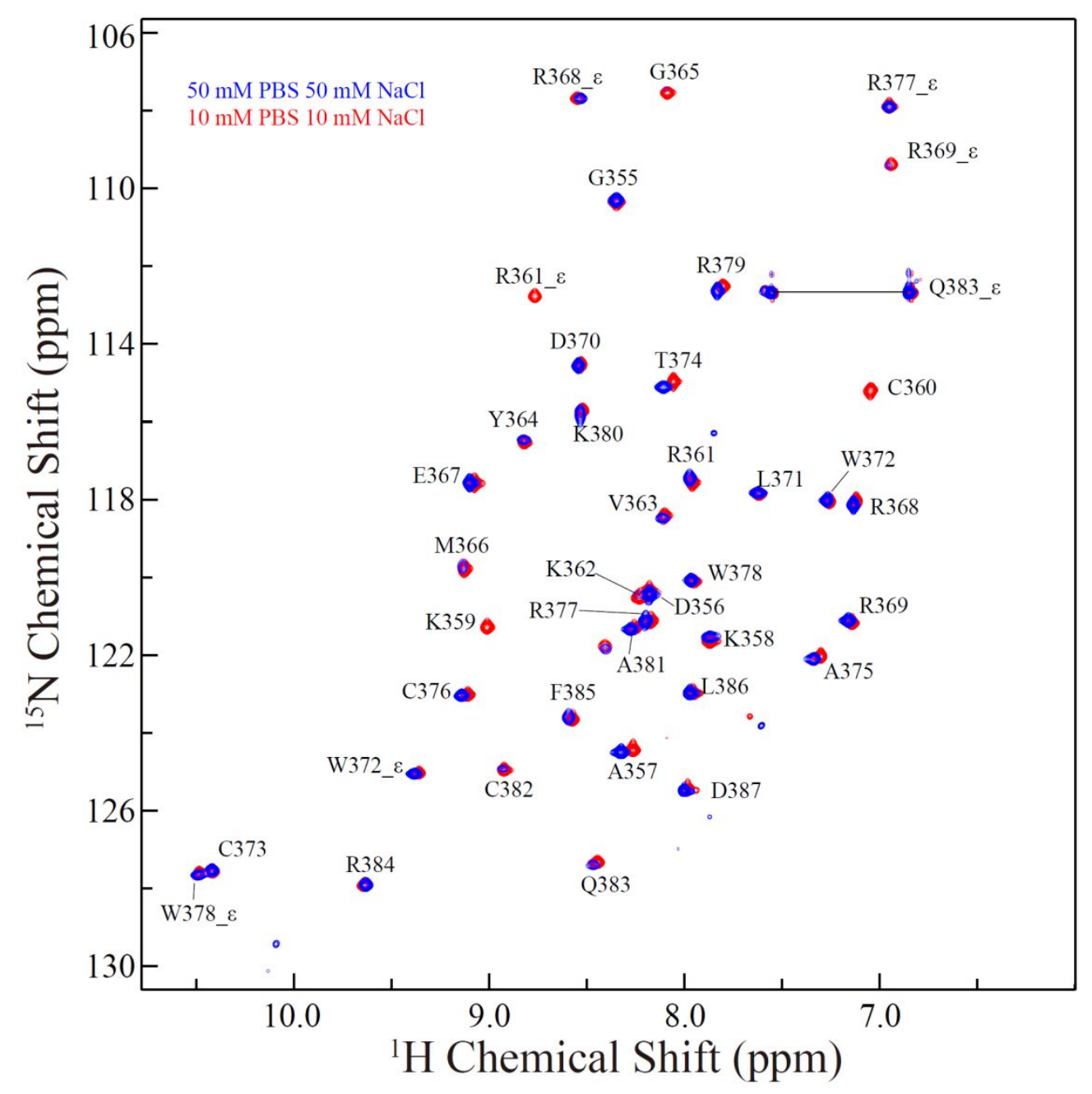

Figure S1 2D ${ }^{1} \mathrm{H}^{-15} \mathrm{~N}$ HSQC spectra of C-clamp ${ }^{\mathrm{HDBP} 1}$ in complex with equimolar ratio of HD5DNA under different buffer conditions. 


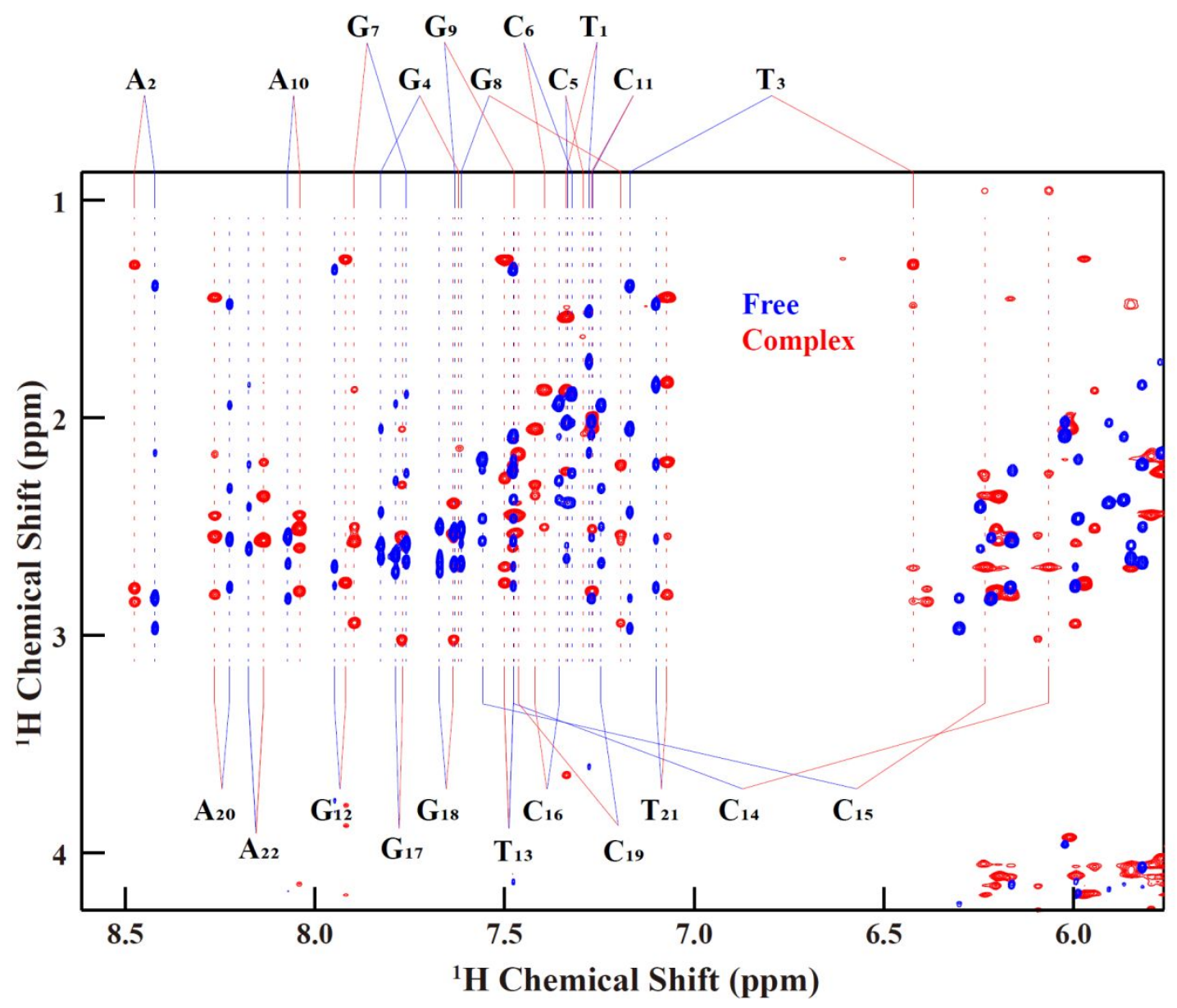

Figure S2 2D F1, F2-15N/13 C-filtered ${ }^{1} \mathrm{H}-{ }^{1} \mathrm{H}$ NOESY spectra of HD5 DNA in its free form (blue) or in complex with equimolar of C-clamp ${ }^{\text {HDBP1 }}$ (red). The straight dashed lines indicate the H6/H8 protons. 
Lys359

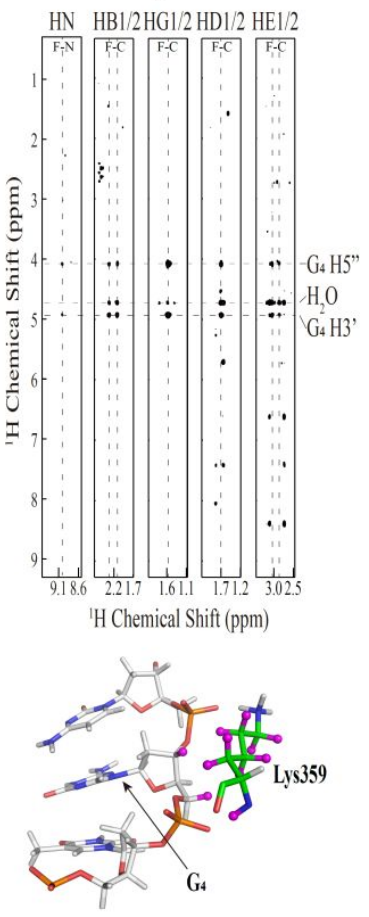

$\operatorname{Arg} 361$
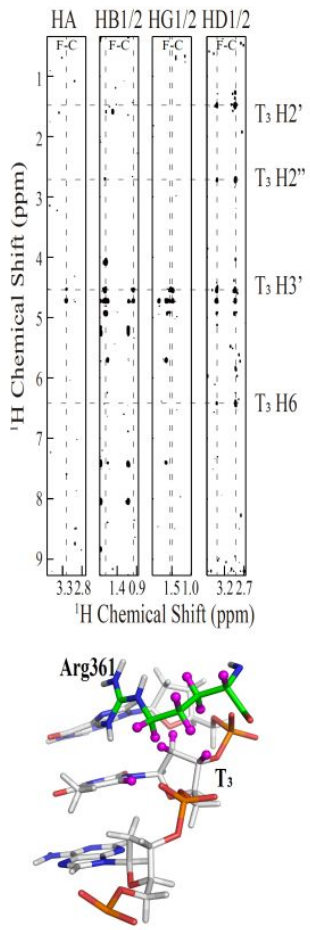

Lys362
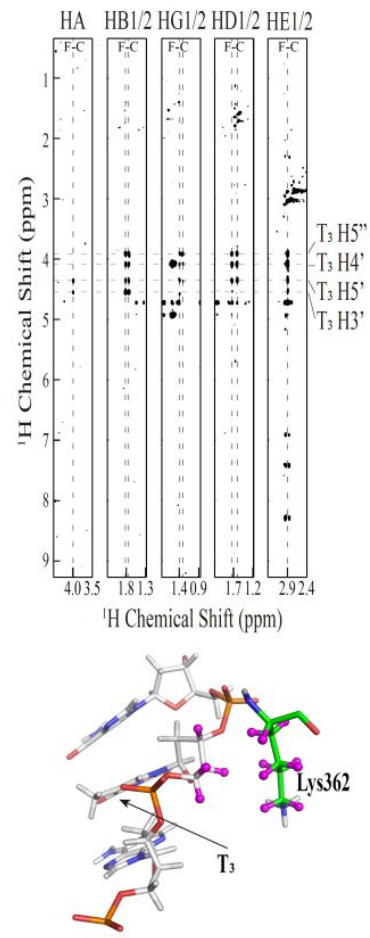

Lys379

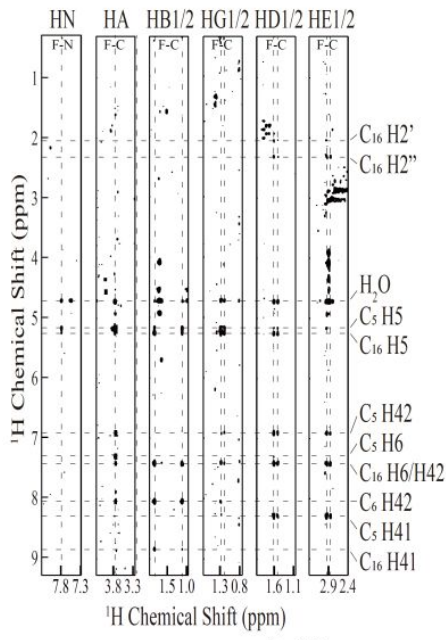

Lys379

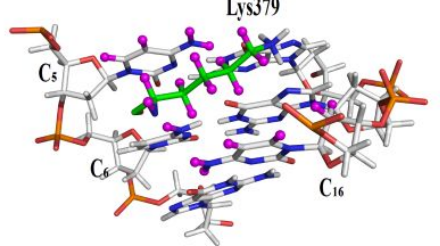

$\operatorname{Trp} 378$
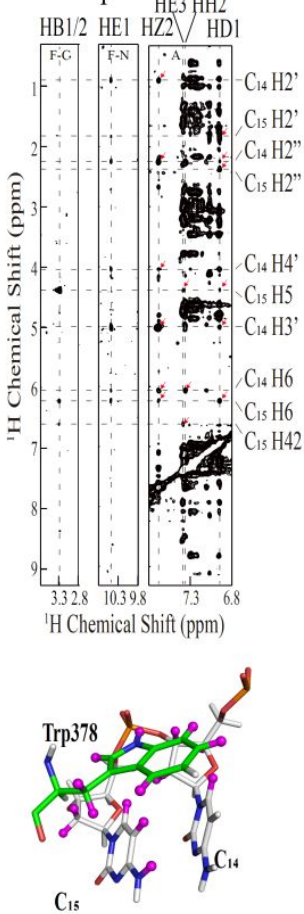

Lys 380

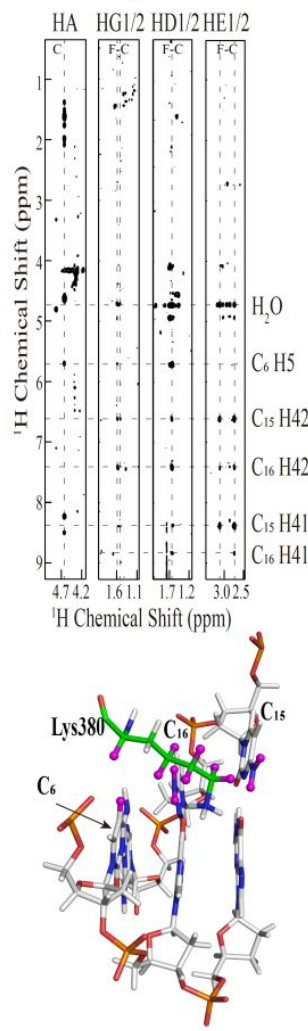

Ala381
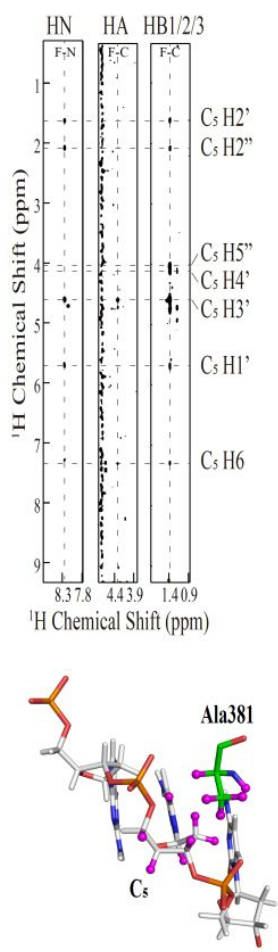

Figure S3 Intermolecular NOE signals between C-clamp ${ }^{\mathrm{HDBP}}{ }^{\text {and HD5-DNA. The related protons }}$ in the complex structure are shown as magenta spheres. F-N: $3 \mathrm{D} F 1-{ }^{15} \mathrm{~N} /{ }^{13} \mathrm{C}$ filtered, $\mathrm{F} 2-{ }^{15} \mathrm{~N}$ edited NOESY-HSQC. F-C: 3D F1- ${ }^{15} \mathrm{~N} /{ }^{13} \mathrm{C}$ filtered, F2-13 $\mathrm{C}$ edited NOESY-HSQC. C: $3 \mathrm{D}{ }^{1} \mathrm{H}-{ }^{13} \mathrm{C}$-edited NOESY-HSQC. A: 2D ${ }^{13} \mathrm{C}$-edited ${ }^{1} \mathrm{H}-{ }^{1} \mathrm{H}$ NOESY optimized for aromatic resonances. 
a

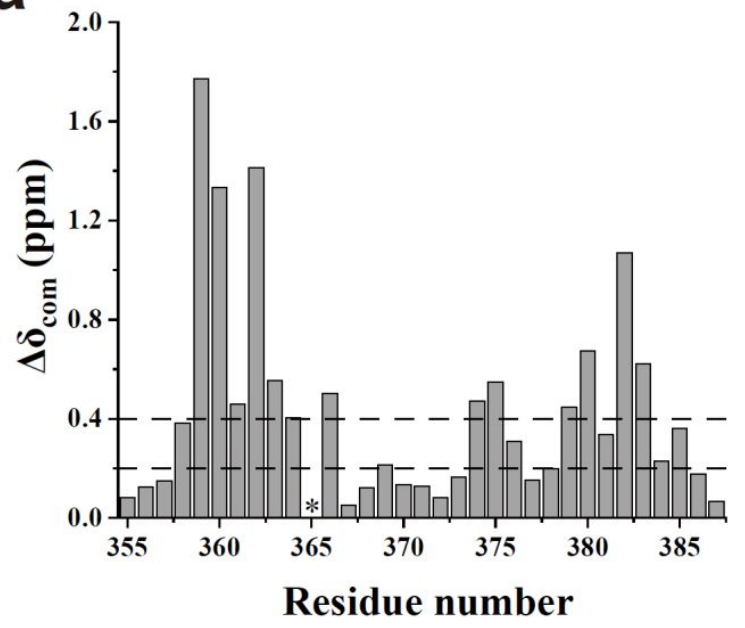

b

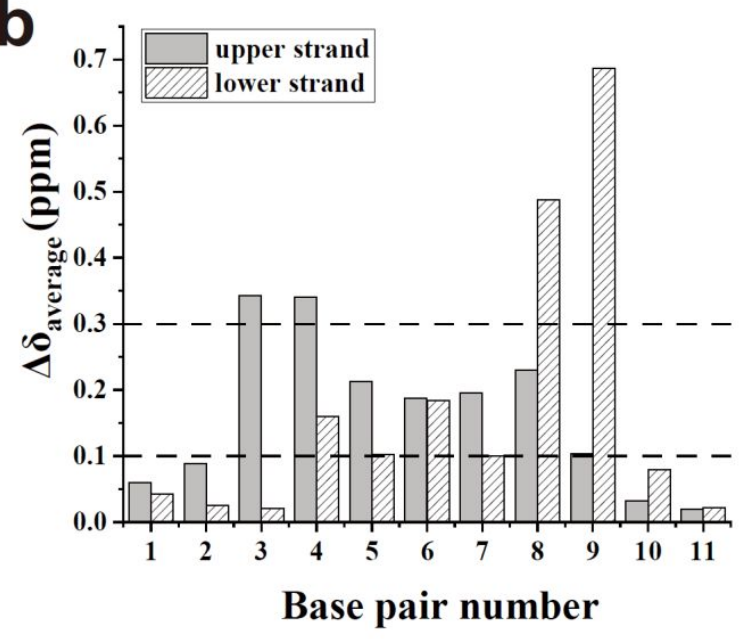

$\Delta \delta \operatorname{com}>0.4$

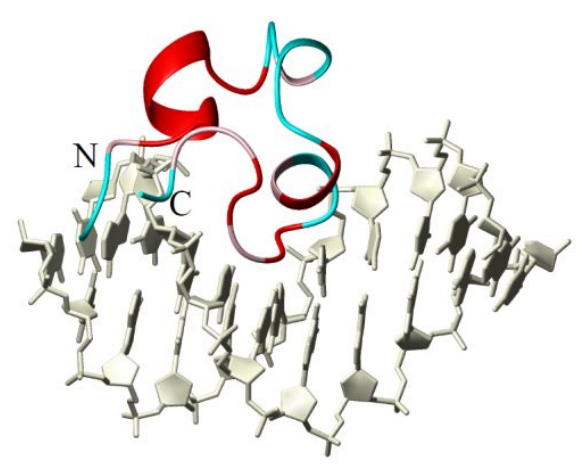

$\Delta \delta_{\text {average }}>0.3$

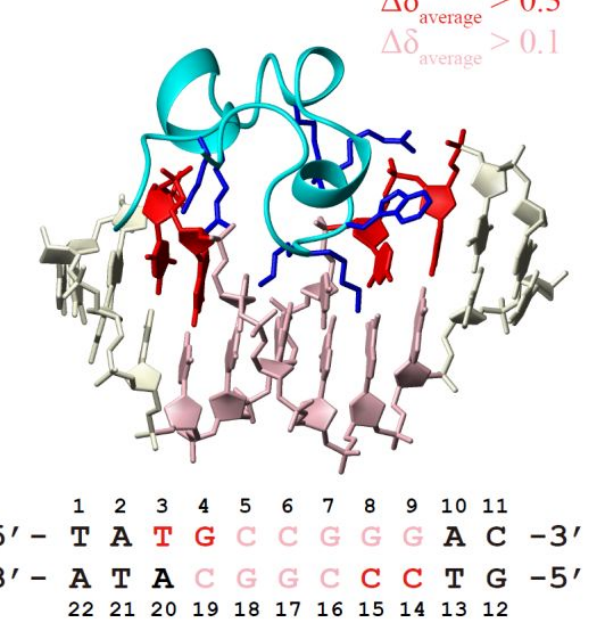

Figure S4 Chemical shift perturbations between C-clamp ${ }^{\mathrm{HDBP} 1}$ and HD5-DNA. a Combined chemical shift $\left(\Delta \delta_{\text {comb }}=\left[\Delta \delta_{\mathrm{HN}^{2}}+\left(\Delta \delta_{\mathrm{N}} / 6.5\right)^{2}\right]^{1 / 2}\right)$ differences of the backbone NH signals of Cclamp ${ }^{\mathrm{HDBP} 1}$ between free and DNA-bound states. The signal of residue G365 is invisible in the free state. b Average absolute chemical shift changes of all resolved DNA protons per nucleotide residue. 

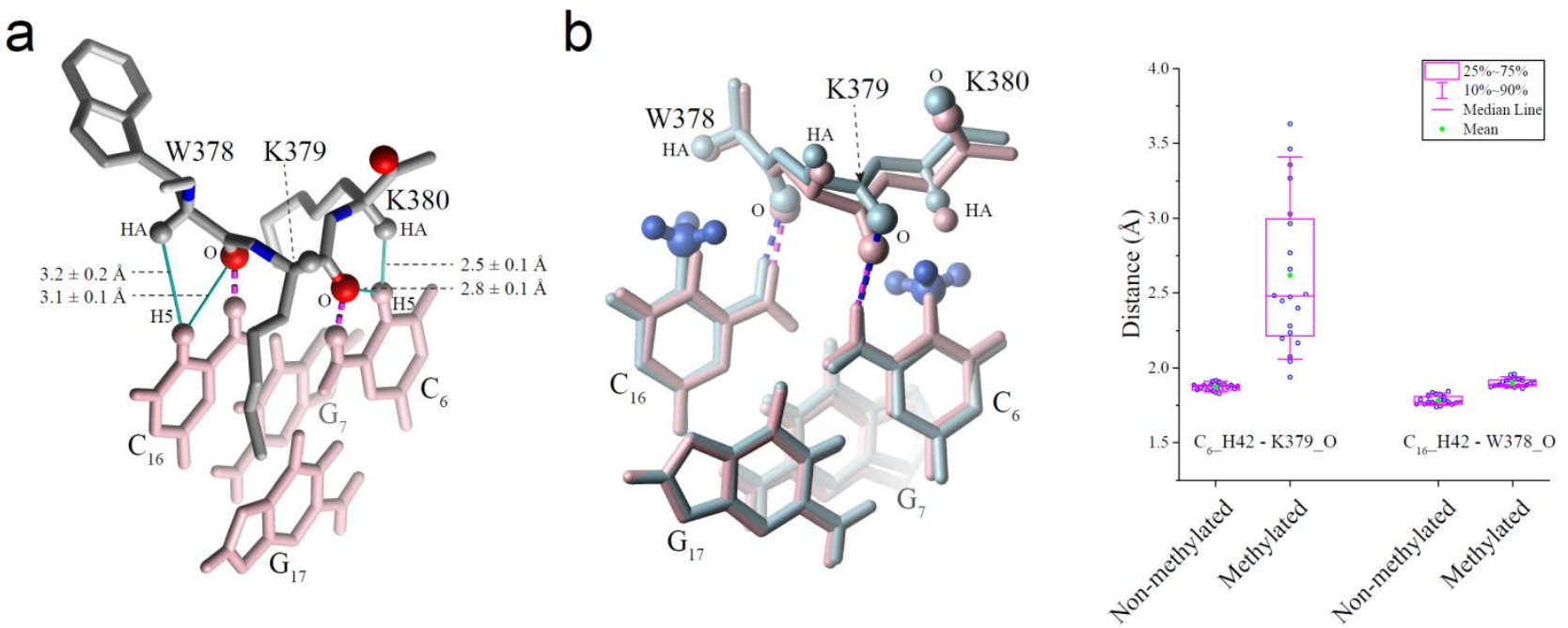

Figure S5. Methylation of the CpG dinucleotide influences the formation of hydrogen bonds between C-clamp ${ }^{\text {HDBP1}}$ and HD5-DNA. a Distances between the cytosine H5 atoms of the CpG dinucleotide and backbone of the "Trp-Lys-Lys" loop in the structure of C-clamp ${ }^{\text {HDBP1}}$ /HD5-DNA complex. b The $\mathrm{C}_{6}$ and $\mathrm{C}_{16}$ bases are substituted by methylated cytosines in the ensemble with 20 structures, which were then subjected to energy minimization using AMBER. A structure model with methylated $\mathrm{CpG}$ (light blue) and the original structure with non-methylated $\mathrm{CpG}$ (pink) are compared on the left, and distances between the $\mathrm{H} 42$ atoms of $\mathrm{C}_{6} / \mathrm{C}_{16}$ and the carbonyl groups of $\mathrm{K} 379 / \mathrm{W} 378$ after energy minimization are shown on the left. 


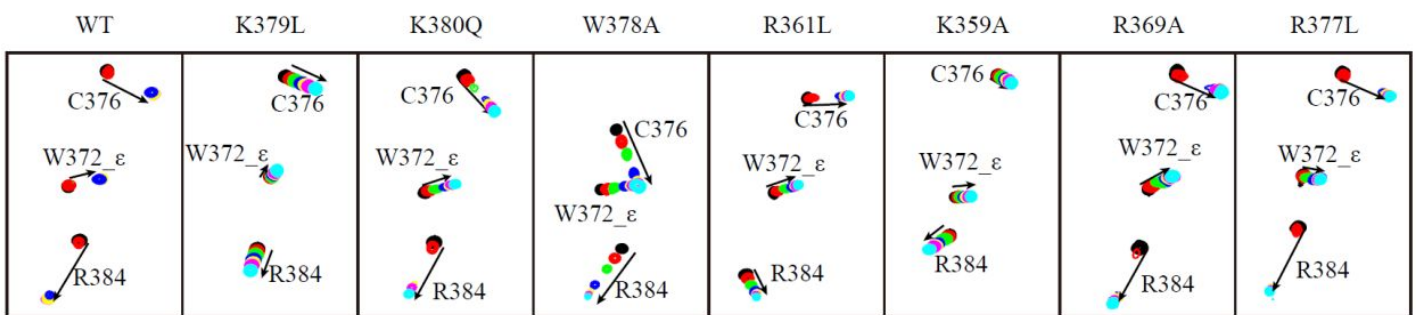

Figure S6 NMR titration experiments of HD5-DNA to wild-type (WT) or different single residue mutants of C-clamp ${ }^{\mathrm{HDBP} 1}$. The regions of the $2 \mathrm{D}{ }^{1} \mathrm{H}-{ }^{15} \mathrm{~N}$ HSQC spectra containing the $\mathrm{NH}$ signals of Cys376 and Arg384, and the side chain $\varepsilon-\mathrm{NH}$ signal of Trp372 are shown.

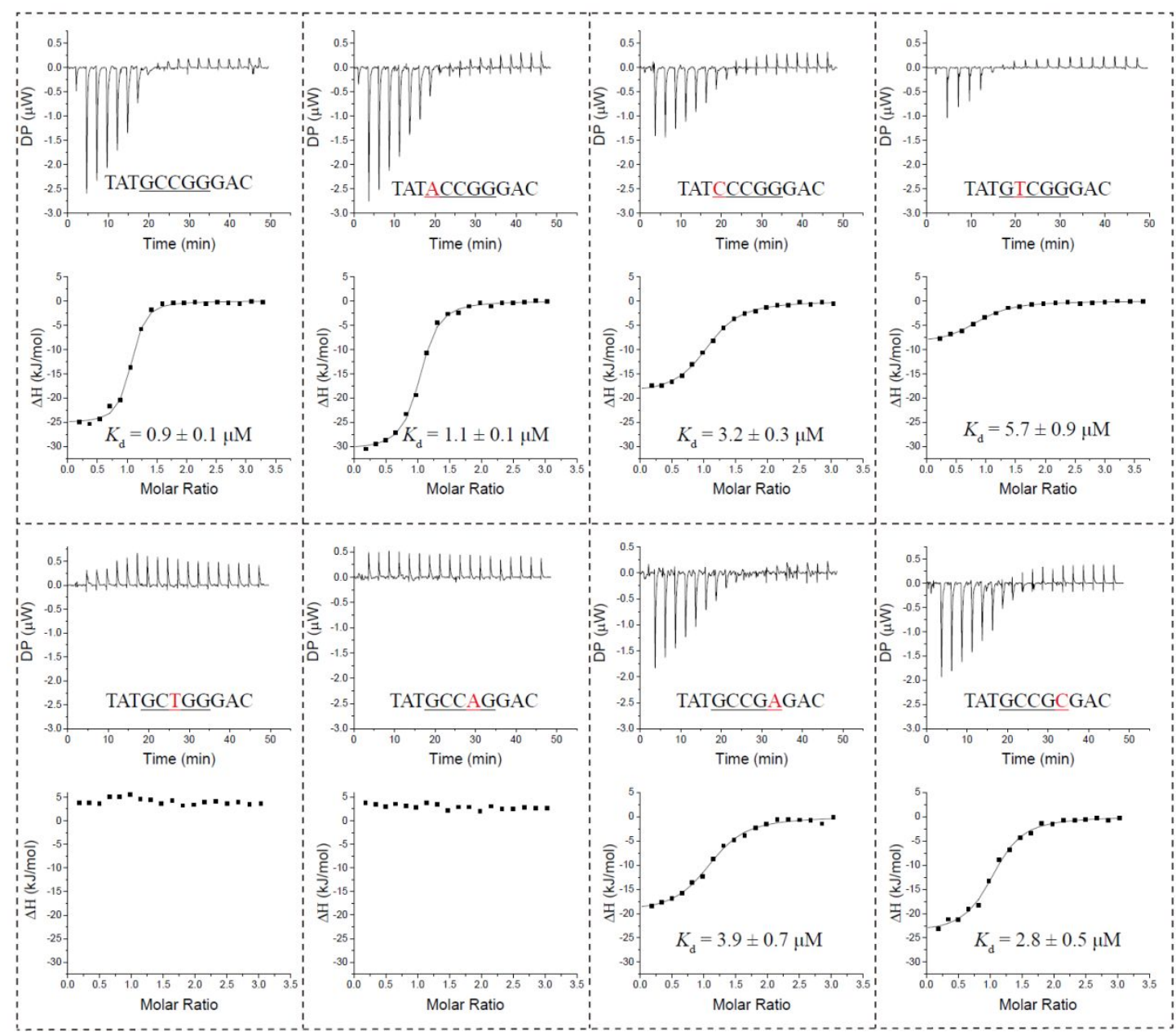

Figure S7 Representative ITC titration curves that measure the binding affinities of different DNA molecules with C-clamp ${ }^{\text {HDBP1}}$. The $K_{d}$ values indicate the average of two measurements and the uncertainties indicate the combined standard deviations. 


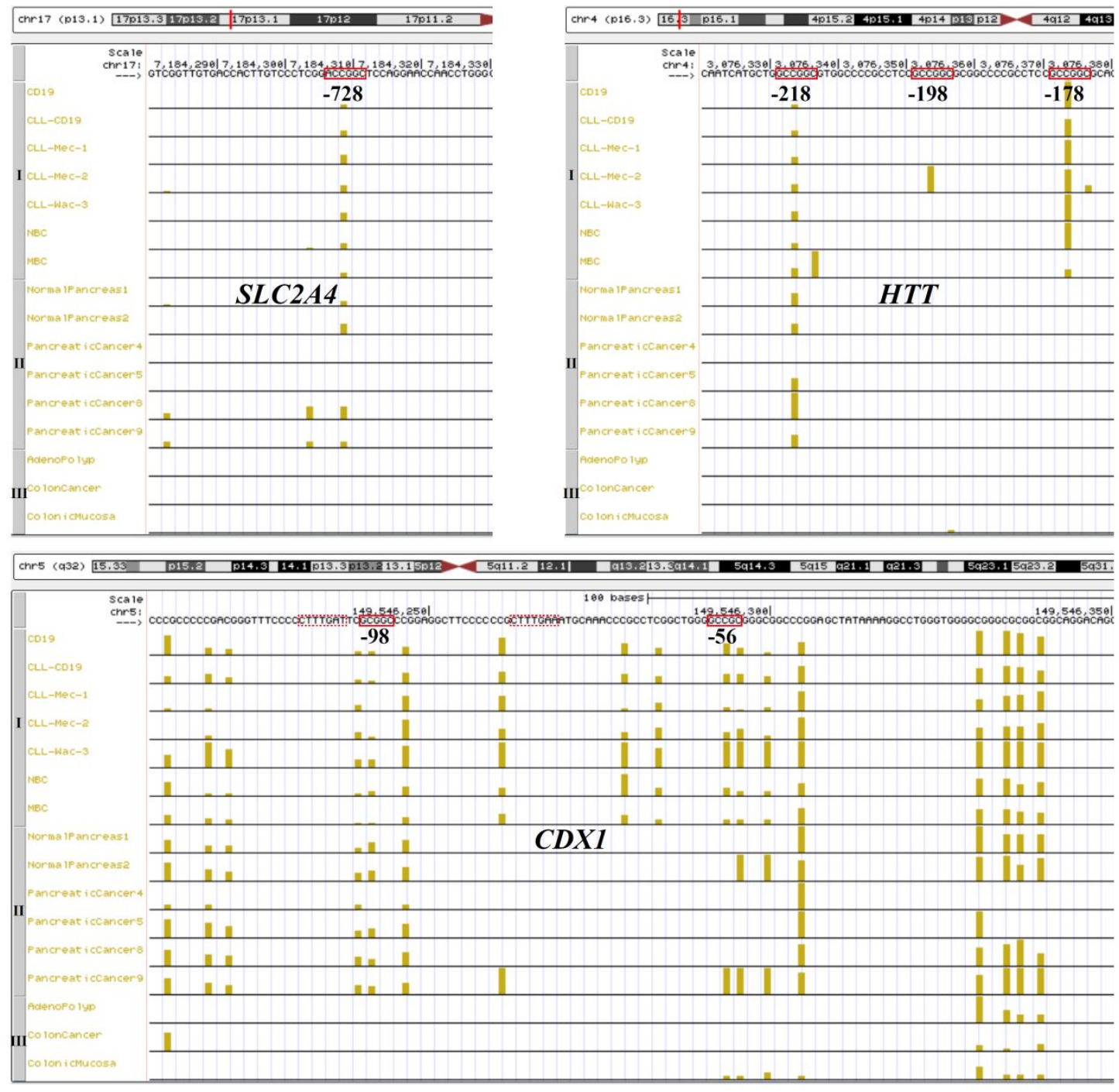

Figure S8 Examples of the methylation states of the binding sites of HDBP1 around the promoter regions of $S L C 2 A 4$ and $H T T$, as well as the binding sites of TCF1E around the promoter region of $C D X 1$ gene. The red solid boxes indicate the binding sites of the C-clamp domains, and the red dashed boxes indicate the binding sites of the HMG domain of TCF1E. In data set I, CD19, NBC, and $\mathrm{MBC}$ represent normal $\mathrm{CD}^{+} 9^{+}, \mathrm{CD}_{19} / \mathrm{IgD}^{+}$naïve, and CD19+/CD27+ memory, respectively ${ }^{1}$. CLLCD19 represent CD19+ $\mathrm{B}$ cell from CLL patients. CLL-Mec-1, CLL-Mec-2, CLL-Wac-3 are three CLL cell lines. Data set II are from nonmalignant pancreas and pancreatic ductal adenocarcinomas tumors $^{2}$. Data set III are from adenomatous polyps, colorectal cancers, and normal colonic mucosa ${ }^{3}$. 


\section{REFERENCES}

1 Pei L, Choi JH, Liu J et al. Genome-wide DNA methylation analysis reveals novel epigenetic changes in chronic lymphocytic leukemia. Epigenetics 2012; 7:567-578.

2 Thompson MJ, Rubbi L, Dawson DW, Donahue TR, Pellegrini M. Pancreatic cancer patient survival correlates with DNA methylation of pancreas development genes. PloS one 2015; 10:e 0128814.

3 Hansen KD, Timp W, Bravo HC et al. Increased methylation variation in epigenetic domains across cancer types. Nature Genetics 2011; 43:768-775. 